LA-UR-01-3392

Approved for public release; distribution is unlimited.

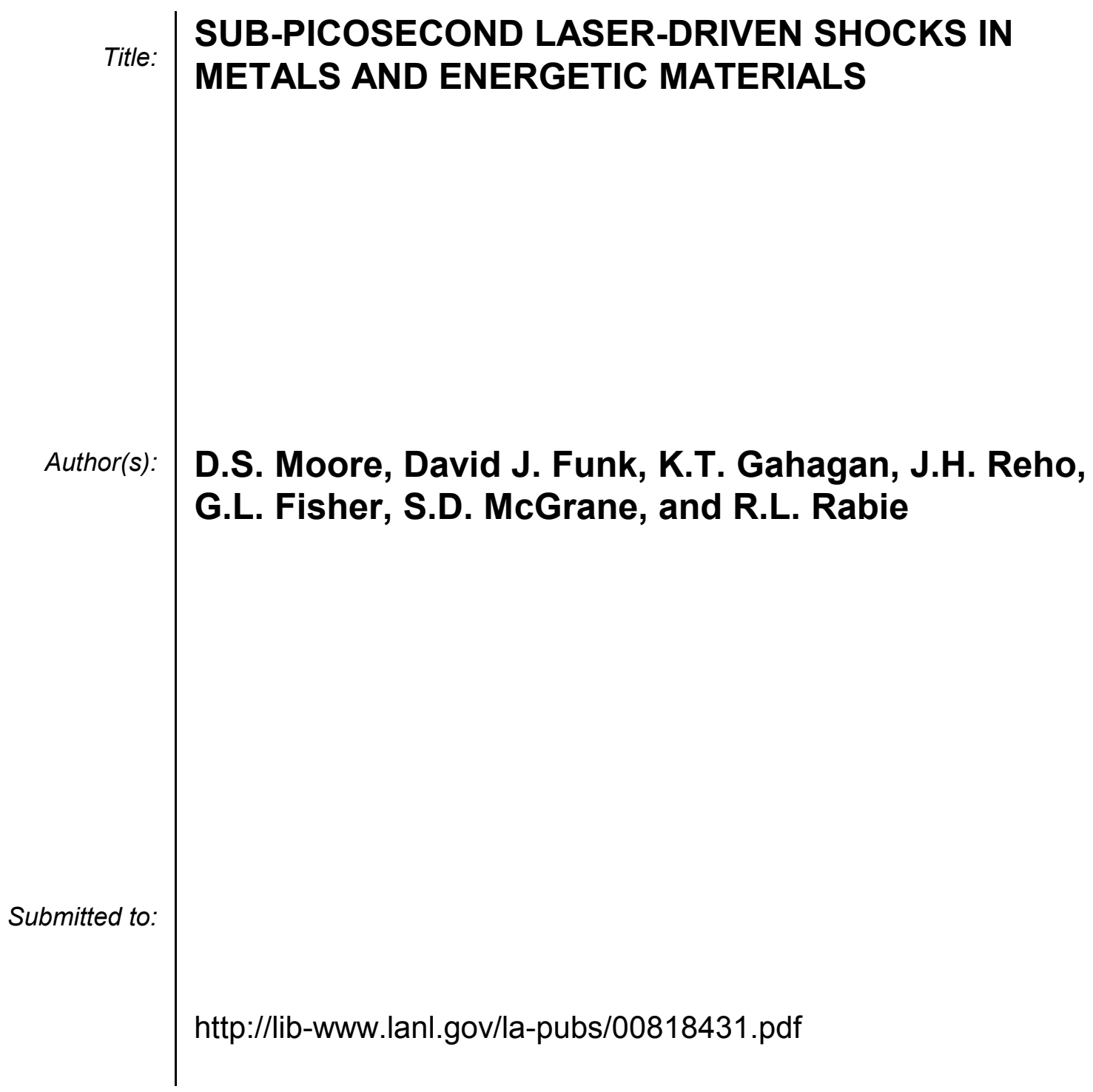




\title{
SUB-PICOSECOND LASER-DRIVEN SHOCKS IN METALS AND ENERGETIC MATERIALS
}

\author{
D.S. Moore ${ }^{1}$, David J. Funk ${ }^{1}$, K.T. Gahagan ${ }^{2}$, J.H. Reho ${ }^{1}$, \\ G.L. Fisher ${ }^{1}$, S.D. McGrane ${ }^{1}$, and R.L. Rabie ${ }^{1}$ \\ ${ }^{1}$ Dynamic Experimentation Division, Los Alamos National Laboratory, Los Alamos, NM 87545 USA \\ ${ }^{2}$ Corning Inc., NYSP-FR-18, Corning, NY 14831 USA
}

\begin{abstract}
A high-energy sub-picosecond laser was used both to drive a shock into thin film targets and to spectroscopically interrogate the shocked material. Targets were thin films of molecular materials coated or grown upon thin vapor-plated metal films on thin glass substrates, or neat metal films on thin glass substrates. The non-linear optical interaction of the shock-driving laser with the thin glass substrate produced surprisingly flat shock waves. Sub-picosecond time-resolved frequency- and spatial-domain interferometries were used to characterize the shock wave as it transited from the thin metal film into the thin molecular material layer. Overviews of the effect of the pressure-dependent complex index of refraction of the shocked thin film metal layer, ultrafast interferometric interrogation of shocked molecular materials (examples: glycidyl azide polymer and nitrocellulose thin films), and progress in preparation of, as well as the need for, uniform, well oriented, thin energetic material layers appropriate to such highly time-resolved methods are presented.
\end{abstract}

\section{INTRODUCTION}

HERCULES (high explosive reaction chemistry via ultrafast laser excited spectroscopies) is a project at Los Alamos National Laboratory begun in 1998 to utilize recent advances in ultrafast laser technology and thin film sample preparation to try to unravel the details of chemical reactions induced in energetic materials by strong shock waves. Using a portion of the same ultrafast high energy laser pulse to drive the shock wave, diagnose the shock state, and spectroscopically probe the shocked sample, drastically reduces the "time jitter" problem associated with traditional shock wave techniques to sub-picosecond levels, sufficient to time resolve many of the ultrafast processes that occur.

The questions HERCULES hopes to answer are: 1) Is the initiating shock impulsive or more gradual (i.e., what is the rise time of the shock wave stress)? 2) Is a process like multi-phonon up-pumping necessary to excite reaction? 3) Are the observed kinetics in accord with standard transition state theory? 4) Are the reactants and/or products ever in thermodynamic equilibrium on time scales important to the usual HE applications? and 5) Is non-equilibrium temperature (vibrationally hot products) essential to sustaining a detonation wave?

This paper will briefly outline the experimental methods used to produce and characterize the laser induced shocks, both in thin metal films, and in samples composed of energetic materials coated on top of thin metal films. The methods and results are discussed in more detail in other conference papers, to which the reader will be pointed. The implications of the results to date will then be discussed.

\section{EXPERIMENTAL}

The entire experimental apparatus is shown schematically in Fig. 1. The laser pulses used in 
the system are typically $20 \mathrm{~mJ}$ at $10 \mathrm{~Hz}$ repetition rate, $800 \mathrm{~nm}$ wavelength, and $130 \mathrm{fs}$ pulse length, produced by a chirped pulse amplified Ti:sapphire laser system (Spectra Physics). Beam splitters and neutral density filters (absorbing) are used to control the pulse energies in the shock drive, interferometry, and spectroscopic interrogation beams. The sequence of pulses within an individual shock experiment is shown in Fig. 2. After the shock drive pulse strikes the substrate/metal film interface, laser ablation processes launch a shock, which travels through the metal film and arrives at either the free metal surface or the metal/energetic material interface. An interferometry pulse arrives at some

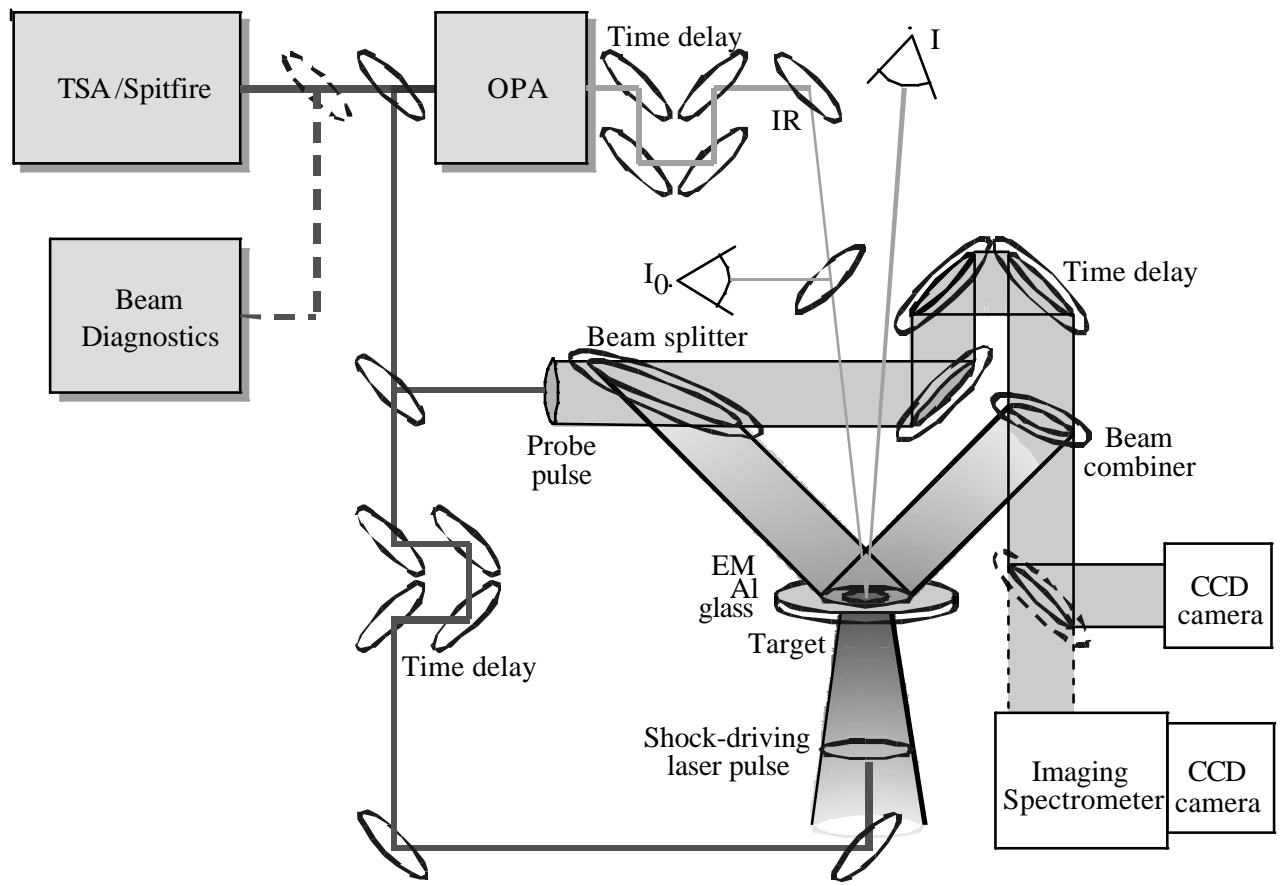

FIGURE 1. Schematic diagram of the ultrafast laser-driven shock experimental apparatus. See text for details.

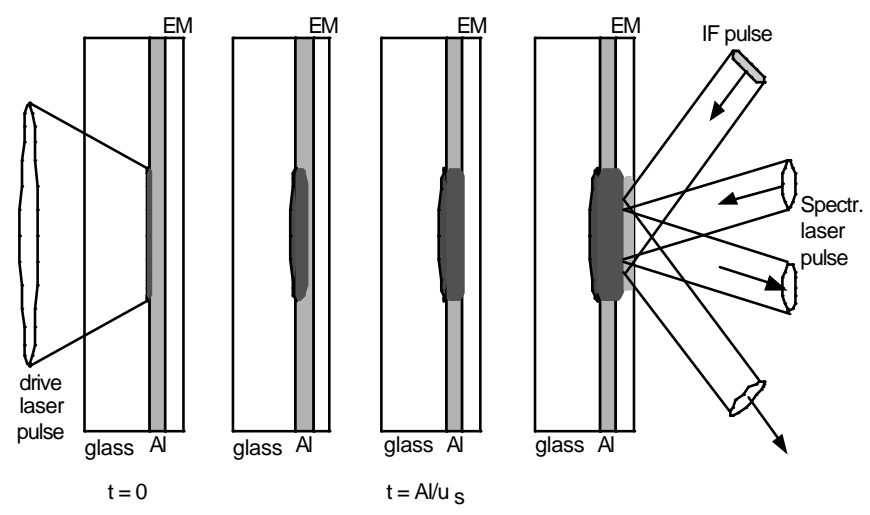

FIGURE 2. Sequence of laser pulses within an individual shock experiment. There are four time snapshots represented: $t=0$ when the drive laser pulse strikes the glass/Al interface, $\mathrm{t}<$ (thickness of $\mathrm{Al}$ )/Us, $\mathrm{t}=$ (thickness of $\mathrm{Al}$ )/Us, and $\mathrm{t}=$ when the shock is mostly through the $\mathrm{EM}$ layer. 
time near when the shock arrives. An optical time delay is used to adjust the relative time of shock breakout and interferometry pulse arrival, in order to build up a time history of shock propagation in step-wise fashion. The target is rastered to a fresh unused spatial location between individual shock experiments. When used, the spectroscopy pulse arrives at the sample at some later time, after the shock has transited an appropriate thickness of overlayer material.

The optical time delays are under computer control to allow several different kinds of time profiles to be recorded. Delaying the shock drive pulse relative to the interferometry pulse results in a record of metal surface motion, and therefore shock state information. Delaying the spectroscopic pulse relative to the shock drive pulse can allow recording of time dependent species concentrations, and therefore kinetics.

Two types of femtosecond (fs) time-resolved interferometry are used. Both utilize a MachZehnder interferometer with the sample in one arm and a time delay in the reference arm. For spectral interferometry (spectral IF, also known as frequency domain interferometry - FDI), the reference arm is slightly longer than the sample arm and the beams are recombined collinearly and focused into a spectrometer. The two probe pulses are stretched in time by the spectrograph, so that they overlap in time and space at the CCD and produce an interference pattern along the wavelength axis. The resulting CCD image for each experiment is analyzed using Fourier transform methods [1-3] to extract the difference in phase between the two probe pulses caused by changes in the film surface position or the optical properties of the metal film. In order to extract the relative contributions of motion and optical properties, data are recorded at two different incidence angles, one near normal incidence and one near the quasi-polarizing angle. Further details and results for several metals can be found in Funk, et al., (Reference 4 and also in this proceedings volume [5]).

For fs time-resolved 2-D spatial interferometry (spatial IF), the reference and sample arms of the interferometer are made the same length, and the beams are combined non-collinearly, so that an interference pattern is produced at the CCD (no spectrograph is used). Two dimensional spatial information about material motion and/or sample optical properties is obtained from the interference patterns using 2-D Fourier transform methods. Again, two different angles of incidence of the sample beam are used to extract the relative contributions of the two effects.

This new method for measuring the shock state of materials with ca. 100 fs time resolution, provides an extraordinary amount of information, and is discussed in detail by Gahagan, et al., in this proceedings volume [6].

Our early ultrafast laser driven shock experiments in thin metal films showed some surprising features. First, the spectral IF data indicated that the shock wave exiting the free metal surface, under a range of drive laser energies, was flat instead of Gaussian in profile, even though the drive laser spatial profile was Gaussian. Serendipitously, the drive laser was focused through the substrate (borosilicate glass microscope slide cover slips of ca. $150 \mu \mathrm{m}$ thickness) before interacting with the glass-metal interface. Turning the sample around so that the drive laser struck the metal film first and observing the shock as it passed from the metal into the substrate resulted in a Gaussian spatial profile. Apparently, interactions of the femtosecond laser pulse with the substrate material flattened the laser intensity spatial profile, which was confirmed by a series of experiments in uncoated substrates of a variety of materials and thicknesses [see Reference 7]. The planarity of the resulting shock waves is astonishing, measured at $<1 \mathrm{~nm}$ RMS over a $75 \mu \mathrm{m}$ diameter, and ensures subsequent material motion, optical properties, and chemical reaction data are obtained under 1-D strain conditions.

\section{RESULTS}

\section{Metal optical properties}

The spectral IF (800 nm wavelength and ppolarization) data obtained from a sample of vapor plated aluminium on a borosilicate glass cover slip 

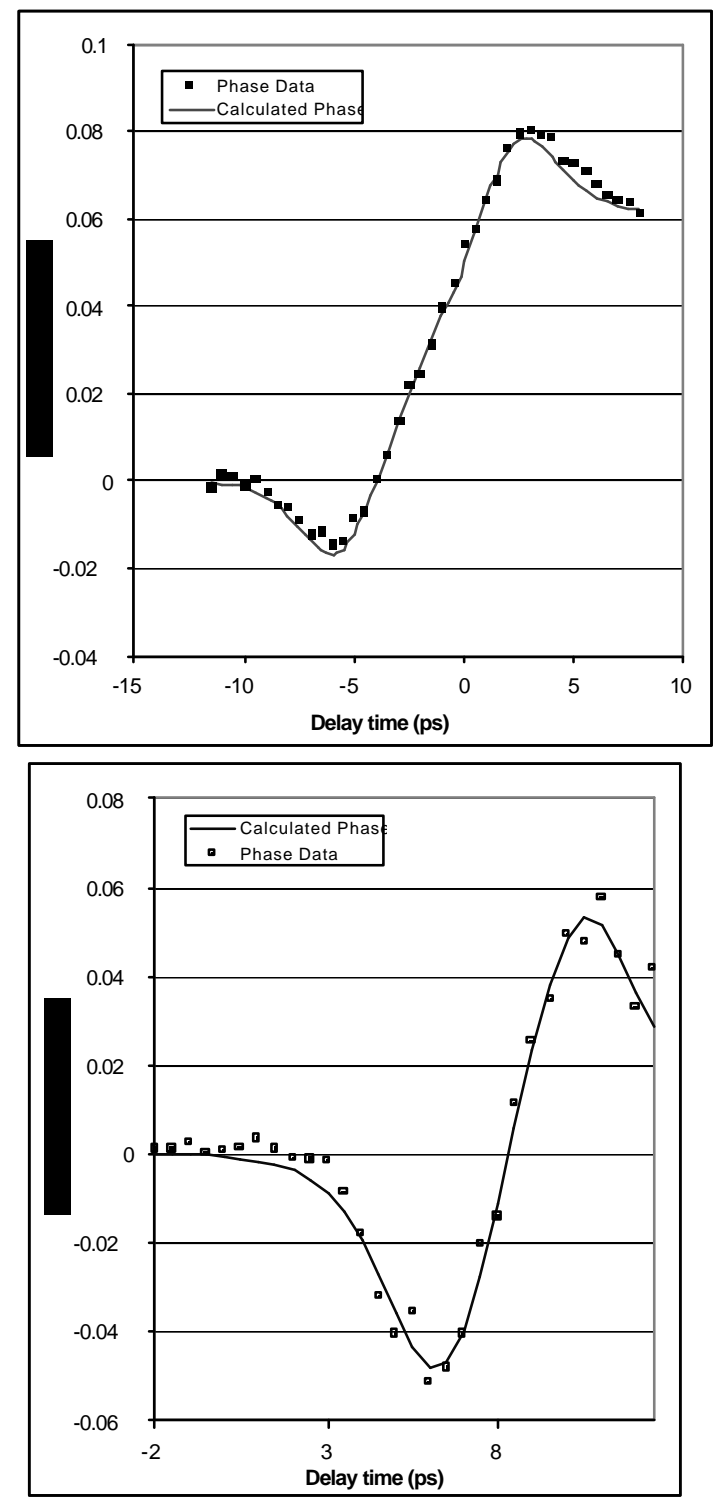

FIGURE 3. Phase shift measured by spectral IF as the shock wave exits the free surface of a $750 \mathrm{~nm}$ thick $\mathrm{Al}$ layer on borosilicate glass substrate. Probe was p-polarization, $800 \mathrm{~nm}$, and the angle of incidence was top) $32.6^{\circ}$, and bottom) $82.5^{\circ}$. The large negative phase shift is associated with the pressure dependence of the $1.5 \mathrm{eV}$ interband transition in $\mathrm{Al}[4,5]$.

substrate as the ultrafast laser driven shock exited the free surface at two different angles of incidence are shown in Fig. 3. These data show an unexpected phase shift during the shock breakout, opposite in sign to that expected (and observed at later times) from material motion. Further spectral IF measurements using $400 \mathrm{~nm}$ probe wavelength confirm that this phase shift can be associated with the pressure dependence of the $1.5 \mathrm{eV}$ interband transition in aluminium. For details see References 4 and 5.

This effect has been seen in all metal samples studied to date, although more usually the phase difference caused by the optical properties is of the same sign as the motion. However, making measurements at two or three angles of incidence allows separation of the contributions and yields an effective complex index of refraction for the shocked material. Use of three angles of incidence is more generally useful, as the separation of the contributions does not require assuming a form for the pressure versus time curve.

\section{Energetic polymers}

Samples for the energetic polymer (EM) studies were produced by spin coating thin polymer films on top of $250 \mathrm{~nm}$ thick Al layers, which had been vapor plated onto borosilicate glass microscope cover slips. These sandwiches were then subjected to ultrafast laser driven shocks, as above. The drive pulse energy was approximately $0.5 \mathrm{~mJ}$ into a focal diameter of ca. $75 \mu \mathrm{m}$, in order to achieve planar shock loading [7] as the shock wave transited from the aluminium into the polymer.

Spatial IF measurements were made of the aluminium/polymer interface, which was assumed to be the reflecting surface before, during, and after the shock transited from the aluminium into, and progressed through, the polymer. This assumption was checked by obtaining data at two probe angles of incidence, where changes in optical properties of the polymer layer behind the shock should be evident.

Figure 4 shows the interface position (obtained from the phase change data using the ambient literature index of refraction) versus delay time obtained from spatial IF measurements in a nitrocellulose layer spin coated on the aluminium, as well as that obtained in a free aluminium surface (no polymer coating) under the same drive laser 
conditions. Similar data are also shown for glycidyl azide polymer films. Details are given in Reho, et al., in this proceedings volume [8]. The nitrocellulose layer was measured by thin film $\mathrm{UV} / \mathrm{vis}$ reflection methods (Filmetrics) to be ca. $550 \mathrm{~nm}$ thick, so that the shock wave has not reached the free nitrocellulose surface during the time delays plotted in Fig. 4.

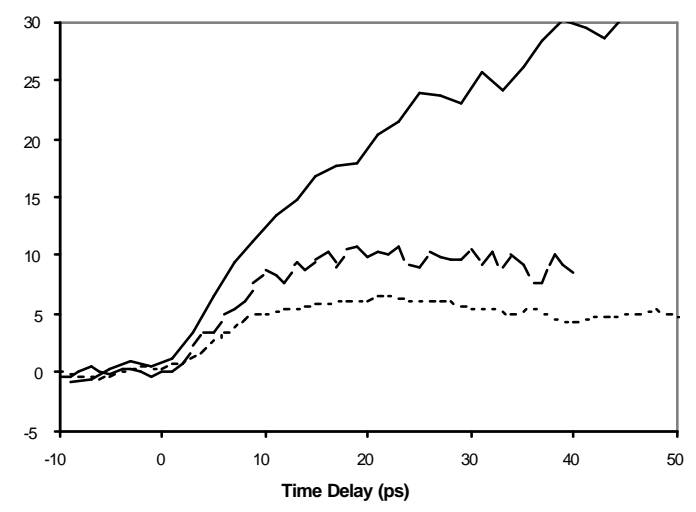

FIGURE 4 Spatial-IF measured $\mathrm{Al}$ surface position versus time delay (zero defined to be when the shock arrives at the $\mathrm{Al}$ rear surface) for three samples: solid) $250 \mathrm{~nm} \mathrm{Al} \mathrm{layer} \mathrm{(no} \mathrm{EM}$ layer), dashed) $250 \mathrm{~nm} \mathrm{Al}$ layer with ca. $1000 \mathrm{~nm}$ GAP layer, and dotted) $250 \mathrm{~nm} \mathrm{Al} \mathrm{layer} \mathrm{with} \mathrm{ca.} 550 \mathrm{~nm}$ nitrocellulose layer. See text for details.

The main feature to be gleaned from the data given in Fig. 4 is the dramatic difference in the motion of the $\mathrm{Al}$ surface when coated with nitrocellulose or GAP. Hydrodynamic simulations of the surface motion were performed in 1-D using an Eulerian-Lagrangian hydrodynamics code. Literature values for the ballistic electron penetration depth, hot electron diffusion distance, and thermal diffusion depth for aluminium [8] were used to estimate an initial effective velocity/temperature profile in the aluminium near the glass-aluminium interface for the hydrodynamic simulations. The Al free surface motion measurements were used as a benchmark, i.e., the peak initial temperature in the ablation region was adjusted so that the simulation most closely matched the experimental data. The resulting curve closely agrees with the free surface Al data in Fig. 4.

That same initial condition was then used in simulations of the aluminium/polymer interface motion. The results are not yet satisfactory, as the simulation predicts larger surface displacements than measured for the nitrocellulose data. However, the $\mathrm{Al} / \mathrm{NC}$ interface deceleration, recession, and then acceleration at later times may be due to catch up of the rarefaction, and the details of that part of the simulation are still in progress. Data taken at higher incidence angles $\left(75^{\circ}\right.$ in air, which is approximately $38^{\circ}$ at the $\mathrm{Al} / \mathrm{NC}$ interface) show a similar displacement trend with time. The reader is directed to Reho et al. in this proceedings volume [9] for further details.

\section{DISCUSSION}

Progress has been made in measuring shock dynamics in thin film samples using fs laser techniques to shock load the sample and interrogate the shock state. Some surprises have been discovered. These include the production of extremely flat shock waves by the non-linear optical interaction of $130 \mathrm{fs}$ duration shock drive laser pulses with the borosilicate glass substrate material in our samples and the observation of pressure induced changes in the optical properties of metals as a laser-driven shock exits the metal surface.

These laser-driven shocks are being used to dynamically load layers of energetic polymers. The fs time-resolved interferometric methods measure the time dependent position of the Al-EM interface, to try to understand the loading dynamics. Time resolved infrared absorption spectroscopy of the loaded EM layer is presently being investigated using 130 fs tunable infrared pulses. The time dependence of the disappearance of reactant IR bands, with hopefully the appearance of product IR bands, will be used to extract chemical kinetics. However, the loading history must be understood first, as laser-driven shocks using the methods discussed above are not steady waves.

We have found that detailed knowledge of the microscopic structure of our samples is critical to understand the loading history as measured using 2D spatial interferometry. Spin coating of polyme rs 
results in optically transparent layers of precisely measurable thickness, but with unknown density or variation of density with distance through the layer. The position versus time data given in Fig. 4 for GAP and NC films could possibly be explained by density variability through the depth of the coating layer or catch up of the rarefaction in the aluminium driver layer, but it could also indicate reaction. Infrared absorption spectroscopic experiments as well as wave profile measurements versus layer thickness, using a thin vapor plated $\mathrm{Al}$ layer on top of the EM layer (both experiments in progress) will help elucidate the actual situation. In addition, we are obtaining spatial interferometric data at three angles of incidence to extract the timedependent optical properties from the interface motion, and using standard ellipsometry to measure the ambient thin film optical properties and their variation with depth.

Ideally, the thin film samples should be of oriented single crystal material. We are presently working to grow such materials using lattice matching methods and vapor or chemical deposition. Standard surface analytical methods will be used to fully characterize the materials before the shock experiments. Such samples should help alleviate most of the uncertainties such as those seen in our NC and GAP experiments to date.

\section{ACKNOWLEDGEMENT}

This research was performed under the auspices of the US Department of Energy.

\section{REFERENCES}

1. Gahagan, K.T., Moore, D.S., Funk, D.J., Rabie, R.L., Buelow, S.J., and Nicholson, J.W., Phys. Rev. Lett. 85, 3205 (2000).

2. Tokunaga E., Terasaki A., and Kobayashi T., Opt. Lett. 17, 1131 (1992).

3. Geindre J.P., Audebert P., Rousse A., Falliès F., Gauthier J.C., Mysyrowicz A., Dos Santos A., Hamoniaux G., and Antonetti A., Opt. Lett. 19, 1997 (1994).

4. Funk, D.J., Moore, D.S., Gahagan, K.T., Buelow, S.J., Reho, J.H., Fisher, G.L. and Rabie, R.L., Phys Rev B (in press 2001).

5. Funk, D.J., Moore, D.S., Reho, J.H., Gahagan, K.T., McGrane, S.D., and Rabie, R.L., in this volume.

6. Gahagan, K.T., Reho, J.H., Moore, D.S., Funk, D.J., and Rabie R.L., in this volume.

7. Moore, D.S., Gahagan, K.T., Reho, J.H., Funk, D.J., Buelow, S.J., Rabie, R.L., and Lippert, T., Appl. Phys. Lett. 78, 40 (2001).

8. Hohlfeld, J., Wellershoff, S.-S., Güdde, J., Conrad, U., Jähnke, V., and Matthias, E., Chem. Phys. 251, 237 (2000).

9. Reho, J.H., Moore, D.S., Funk, D.J., Fisher, G.L., McGrane, S.D., and Rabie, R.L., in this volume. 